\title{
Sequential Catalysis for Two Remote Asymmetric Reactions
}

\section{Key words}

palladium catalysis

rhodium catalysis

diastereodivergent

synthesis

\section{Synica}

of the Month

17 examples, up to $78 \%$ yield of a single diastereomer in $99 \%$ ee

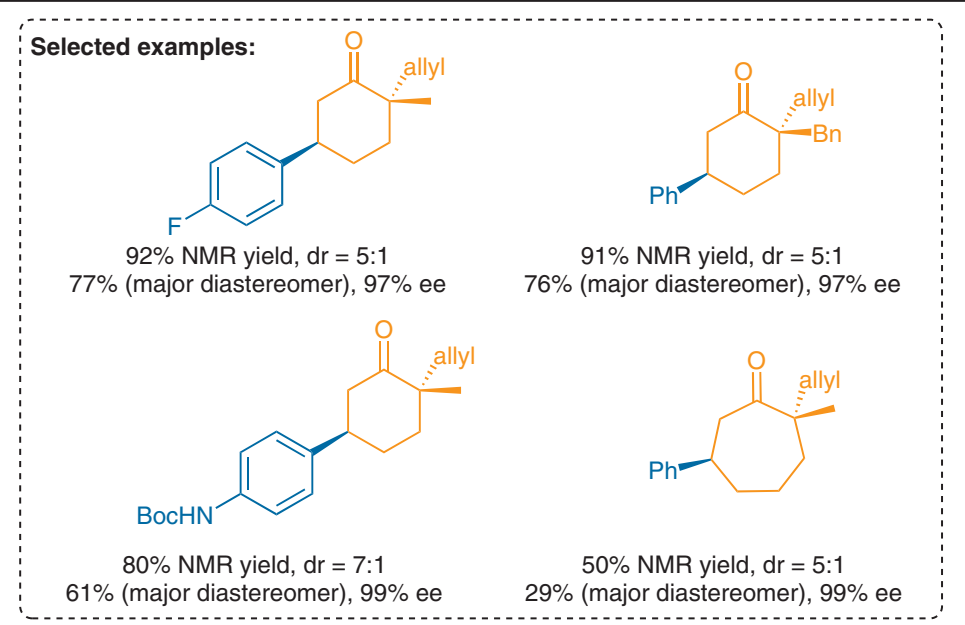

Stereodevergent synthesis:

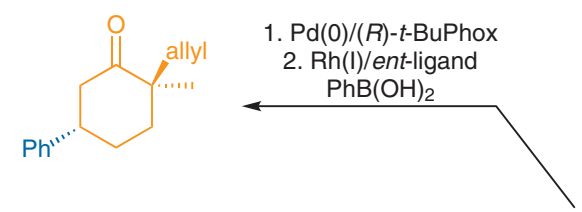

91\% NMR yield, $d r=6: 1$ 73\% (major diastereomer), 99\% ee

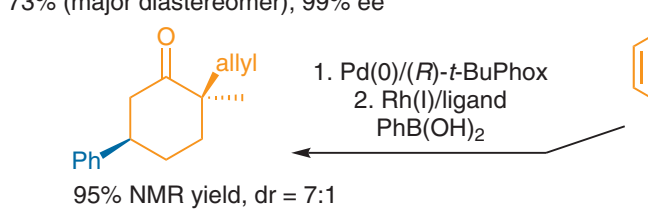

$69 \%$ (major diastereomer), $99 \%$ ee
$\left[\mathrm{Rh}\left(\mathrm{C}_{2} \mathrm{H}_{2}\right)_{2} \mathrm{Cl}\right]_{2}(4 \mathrm{~mol} \%)$

ligand $(8.8 \mathrm{~mol} \%)$ $\operatorname{ArB}(\mathrm{OH})_{2}(1.5$ equiv $)$

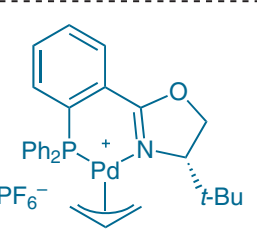

[Pd(S-t-BuPhox)allyl]PF

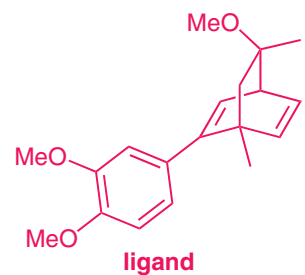

ligand
Significance: The authors report enantio- and diastereodivergent synthesis of ketones with two remote stereocenters using Pd-catalyzed asymmetric allylic alkylation and Rh-catalyzed 1,4-addition in a sequential manner. Although these reaction were reported previously, additional screening had to be done to find similar reaction conditions and ensure compatibility of catalysts. These reactions independently gave only good enantioselectivities.
Comment: A complete one-pot process in which all reagents are present from the beginning of the reaction underperformed. It was found that too much methanol as well as phenylboronic acid interfered with the Pd-catalyzed asymmetric allylic alkylation, decreasing both conversion and ee. Finally, the process was used to prepare all four stereoisomers of the cyclohexenone in good yield (65-73\%) and excellent enantioselectivity (99\% ee). 